BABAR-CONF-01/99

SLAC-PUB-9139

February, 2002

\title{
Rare Hadronic and Radiative Penguin B Decays at BABAR
}

\author{
Stéphane Willocq \\ Department of Physics \\ University of Massachusetts, Amherst, MA 01003, USA \\ Representing the BABAR Collaboration
}

\begin{abstract}
We report recent results in the study of rare hadronic and radiative penguin decays of $B$ mesons. These results are based on a sample of 23 million $B \bar{B}$ pairs collected by the BABAR Collaboration at the SLAC PEP-II $e^{+} e^{-}$B Factory.
\end{abstract}

Contributed to the Proceedings of the $5^{\text {th }}$ KEK Topical Conference:

Frontiers In Flavor Physics (KEKTC5),

20-22 Nov 2001, Tsukuba, Ibaraki, Japan.

Stanford Linear Accelerator Center, Stanford University, Stanford, CA 94309

Work supported in part by Department of Energy contract DE-AC03-76SF00515. 


\title{
Rare Hadronic and Radiative Penguin B Decays at BABAR
}

\author{
S. Willocq ${ }^{\mathrm{a}}$ representing the BABAR Collaboration

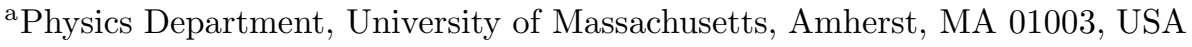

\begin{abstract}
We report recent results in the study of rare hadronic and radiative penguin decays of $B$ mesons. These results are based on a sample of 23 million $B \bar{B}$ pairs collected by the BABAR Collaboration at the SLAC PEP-II $e^{+} e^{-}$ B Factory.
\end{abstract}

\section{MOTIVATION}

A major effort is currently underway to test the flavor sector of the Standard Model (SM) in general and the origin of $\mathrm{CP}$ violation in particular. Measurements of the time-dependent CP-asymmetries in $B^{0} \rightarrow J / \psi K_{s}^{0}$ decays have led to the first observation of $\mathrm{CP}$ violation [1] in the $B^{0}-\overline{B^{0}}$ system and to a measurement of the phase $\beta$ of the Cabibbo-Kobayashi-Maskawa (CKM) matrix element $V_{t d}$-the current world average is $\sin 2 \beta=0.79 \pm 0.10$. The magnitude of $V_{t d}$ is constrained by studies of $B^{0}$ and $B_{s}^{0}$ mixing and is expected to be determined with an uncertainty of $\sim 5 \%$ in the near future.

The determination of the magnitude and phase $(\gamma)$ of the CKM element $V_{u b}$ is expected to be much more difficult, due in part to the small rate for $b \rightarrow u$ transitions $(\sim 0.5-1 \%$ compared to the rate for $b \rightarrow c$ transitions). In parallel with the measurement of $\sin 2 \beta$ with $B^{0} \rightarrow J / \psi K_{s}^{0}$ decays, one can in principle determine $\sin 2 \alpha$ from the time-dependent asymmetry in $B^{0} \rightarrow \pi^{+} \pi^{-}$decays (where $\alpha=\pi-(\beta+\gamma)$ ). However, the study of charmless two-body $B$ decays has shown that gluonic penguin $b \rightarrow d$ processes contribute significantly to the overall $B^{0} \rightarrow \pi^{+} \pi^{-}$decay rate and have a different weak phase than the dominant $b \rightarrow u$ tree process, thereby preventing a straightforward interpretation of the measured asymmetry. Improved understanding of gluonic penguins is thus of very high importance and constitutes an important challenge for current experiments.
Another motivation to study rare hadronic and radiative penguin decays is that many of these decays proceed via effective flavor-changing neutral current (FCNC) transitions. Such FCNC processes are forbidden at tree level and involve heavy particles in loops (e.g. $W$ boson or top quark). This implies that the rates are low but also that such processes are sensitive to the presence of heavy non-SM particles in the loops.

Besides the measurement of decay rates, one can also study $\mathrm{CP}$ violation in the decay (a.k.a. direct $\mathrm{CP}$ violation). In this case, the relevant observable is

$A_{C P}=\frac{\Gamma(\bar{B} \rightarrow \bar{f})-\Gamma(B \rightarrow f)}{\Gamma(\bar{B} \rightarrow \bar{f})+\Gamma(B \rightarrow f)}$.

If both penguin and tree decay amplitudes contribute to the decay, the asymmetry can be expressed as

$A_{C P}=\frac{2|P||T| \sin \Delta \phi \sin \Delta \delta}{|P|^{2}+|T|^{2}+2|P||T| \cos \Delta \phi \cos \Delta \delta}$,

where $|P|$ and $|T|$ represent the magnitudes of the penguin and tree decay amplitudes, whereas $\Delta \phi$ and $\Delta \delta$ correspond to the difference between the weak and strong phases for the penguin and tree amplitudes, respectively. From this expression it can be seen that $A_{C P}$ is expected to be very small in the SM (less than $1 \%$ ) for penguindominated decays like $B \rightarrow \phi K^{(*)}, K^{0} \pi, K^{*} \gamma$, and thus the observation of large $\mathrm{CP}$ asymmetries in these modes would clearly signal the presence of new physics in the penguin loops. 


\section{EXPERIMENTAL DETAILS}

In the following, we report the results of analyses based on a sample of 23 million $B \bar{B}$ events collected by the BABAR Collaboration at the asymmetric-energy PEP-II $e^{+} e^{-}$collider. This sample corresponds to $20.7 \mathrm{fb}^{-1}$ of $e^{+} e^{-}$annihilation data recorded at the $\Upsilon(4 S)$ resonance. An additional (off-resonance) sample corresponding to $2.6 \mathrm{fb}^{-1}$ recorded $40 \mathrm{MeV}$ below the resonance is also used to study continuum backgrounds. Charged particles are tracked and measured with the combination of a five-layer double-sided silicon microstrip detector and a 40-layer drift chamber immersed in a $1.5 \mathrm{~T}$ solenoidal magnetic field. Surrounding the tracking systems, a Cherenkov ring imaging detector (DIRC) relying on total internal reflection of the Cherenkov light produced in $4.9 \mathrm{~m}$-long quartz bars provides charged particle identification. A Thallium-doped CsI electromagnetic calorimeter is used for photon detection and electron identification. Finally, an instrumented flux return provides muon and $K_{L}^{0}$ identification. Further detail about the BABAR detector is given elsewhere [2].

Most of the analyses presented here attempt to fully reconstruct the $B$ decay. To do so, we rely on the well-known beam energies and exploit kinematical constraints from energy and momentum conservation in the $e^{+} e^{-} \rightarrow \Upsilon(4 S) \rightarrow B \bar{B}$ process. Two mostly uncorrelated kinematical variables are used: the beam-energy substituted mass, $m_{E S}=\sqrt{E_{\text {beam }}^{* 2}-\mathbf{p}_{B}^{* 2}}$, and the energy difference in the $e^{+} e^{-}$center-of-mass system (CMS), $\Delta E=E_{B}^{*}-E_{\text {beam }}^{*}$, where $E_{\text {beam }}^{*}$ and $E_{B}^{*}$ are the energies of the beam and the $B$ meson candidate, and $\mathbf{p}_{B}^{*}$ is the $B$ meson candidate momentum vector, all quantities being defined in the CMS. In some analyses, a kinematical fit is performed on the momenta of the $B$ decay products by imposing $E_{B}^{*}=E_{\text {beam }}^{*}$ and the corresponding energyconstrained mass $m_{E C}$ is used. The $m_{E S}$ resolution is dominated by the beam energy spread and has a typical value of $2.5 \mathrm{MeV}$. The $\Delta E$ resolution is different for each particular final state and ranges between 20 and $70 \mathrm{MeV}$ (the resolution is noticeably worse for modes containing photons or $\left.\pi^{0} \mathrm{~s}\right)$.
With the exception of the $B \rightarrow K^{(*)} \ell^{+} \ell^{-}$analysis, the background from $B \bar{B}$ events is small or negligible for all other analyses. The dominant background from $e^{+} e^{-} \rightarrow q \bar{q}$ continuum events $(q=u, d, s, c)$ is suppressed using event shape and energy flow variables. These variables exploit the fact that $B \bar{B}$ events are much more spherical than continuum events due to the small $B$ momentum $(\sim 340 \mathrm{MeV} / c)$ in the CMS.

Analyses have been optimized and tested using signal and background Monte Carlo samples, as well as off-resonance data and side-band data. The signal region remained hidden during this optimization and validation process.

\section{RADIATIVE PENGUIN DECAYS}

As mentioned earlier, the measurement of $B^{0}$ and $B_{s}^{0}$ oscillation frequencies will soon provide a determination of the ratio $\left|V_{t d} / V_{t s}\right|$ with an uncertainty of $\sim 5 \%$. Radiative penguin decays are also sensitive to the same CKM elements and can provide a complementary determination via the ratio $\Gamma(B \rightarrow \rho \gamma) / \Gamma\left(B \rightarrow K^{*} \gamma\right)$.

A first step toward such a measurement is the determination of the branching fraction for $B \rightarrow K^{*} \gamma$ decays. The analysis proceeds with the selection of high-energy photons with $1.5<E_{\gamma}<$ $4.5 \mathrm{GeV}$ and $2.30<E_{\gamma}^{*}<2.85 \mathrm{GeV}$ in the laboratory and CMS frames, respectively. Photons consistent with originating from $\pi^{0}$ and $\eta$ decays are removed. Photons are further required to be isolated to remove contamination from high-energy $\pi^{0}$ decays. $K^{*}$ candidates are reconstructed in $K^{+} \pi^{-}, K_{S}^{0} \pi^{0}, K^{+} \pi^{0}$ and $K_{S}^{0} \pi^{+}$modes use of DIRC information to select charged kaons. The dominant backgrounds consist of events with photons from initial state radiation or from $\pi^{0}$ and $\eta$ decay. These are suppressed by utilizing event shape variables, the polar angle of the $B$ candidate and the helicity angle of the $K^{*}$ decay.

$B \rightarrow K^{*} \gamma$ candidates are required to satisfy $m_{E S}>5.2 \mathrm{GeV}$ and $-0.2<\Delta E<0.1 \mathrm{GeV}$ (for $K^{+} \pi^{-}$and $K_{S}^{0} \pi^{+}$modes) or $-0.225<\Delta E<$ $0.125 \mathrm{GeV}$ (for $K_{S}^{0} \pi^{0}$ and $K^{+} \pi^{0}$ modes). An unbinned likelihood method is used to extract

${ }^{1}$ Charged conjugate states are implied throughout, unless noted otherwise. 


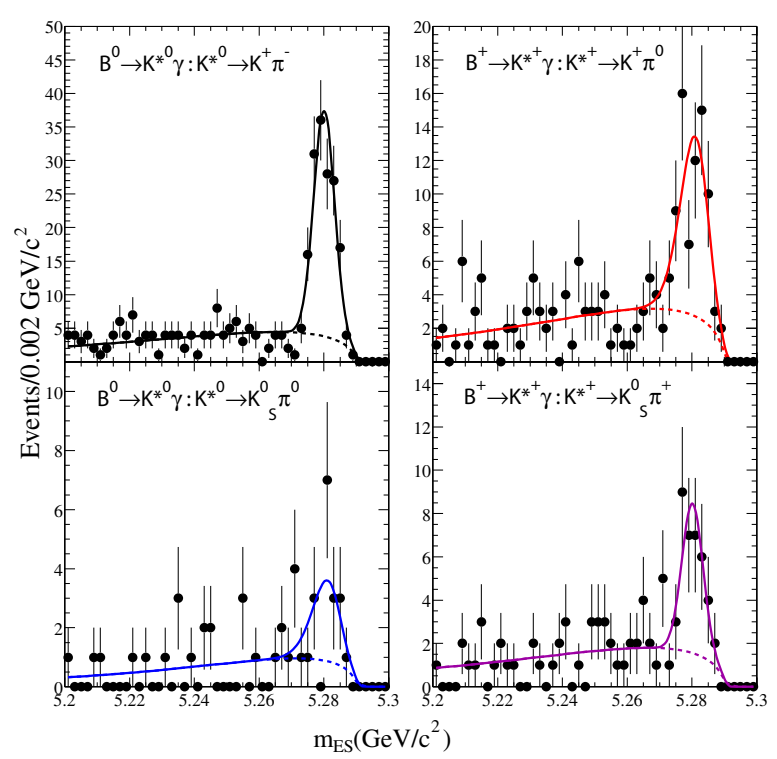

Figure 1. Distribution of $m_{E S}$ for $B \rightarrow K^{*} \gamma$ candidates in four different $K^{*}$ decay channels.

signal yields from the $m_{E S}$ distributions, see Fig. 1. The branching fractions for each of the four modes are shown in Table 1. Averaging the two $K^{* 0}$ and $K^{*+}$ modes yields $\mathcal{B}\left(B^{0} \rightarrow K^{* 0} \gamma\right)=$ $(42.3 \pm 4.0$ (stat) \pm 2.2 (syst) $) \times 10^{-6}$ and $\mathcal{B}\left(B^{+} \rightarrow\right.$ $\left.K^{*+} \gamma\right)=(38.3 \pm 6.2$ (stat) \pm 2.2 (syst) $) \times 10^{-6}$. These values, although somewhat lower, agree with recent theoretical predictions, given the $\sim$ $40 \%$ uncertainty in the predictions.

We have also searched for the rare decay $B^{0} \rightarrow$ $\gamma \gamma$, which is predicted to have a branching fraction in the range of $(0.1-2.3) \times 10^{-8}$ within the SM. However, this could be enhanced by new physics contributing to the loops. Given the current size of the event sample, any observation would be clear evidence for physics beyond the SM. The analysis proceeds in a way similar to the $K^{*} \gamma$ analysis. One event is found in the signal region, defined by the $\pm 2 \sigma$ ranges $\left|m_{E S}-m_{B^{0}}\right|<$ $0.0078 \mathrm{GeV}$ and $|\Delta E|<0.144 \mathrm{GeV}$, in agreement with the expected background of 0.9 event. As a result, a limit is set to be $\mathcal{B}\left(B^{0} \rightarrow \gamma \gamma\right)<$ $1.7 \times 10^{-6}$ at the $90 \%$ C.L. This represents a factor of 20 improvement over the best previous limit.
Table 1

Summary of signal yields and branching fraction measurements for radiative penguin decays. The first and second uncertainties in the branching fractions are statistical and systematic, respectively. The uncertainty in the yield is statistical only.

\begin{tabular}{lcc} 
Mode & Signal yield & $\mathcal{B}\left(10^{-6}\right)$ \\
\hline$K_{K}^{* 0}+\pi^{-} \gamma[3]$ & $135.7 \pm 13.3$ & $42.4 \pm 4.1 \pm 2.2$ \\
$K_{K_{S}^{0} \pi^{0}}^{* 0}$ & $14.8 \pm 5.6$ & $41.0 \pm 17.1 \pm 4.2$ \\
$K_{K_{S}^{0} \pi^{+}}^{* 0}$ & $28.1 \pm 6.6$ & $30.1 \pm 7.6 \pm 2.1$ \\
$K_{K^{+} \pi^{0}}^{*} \gamma$ & $57.6 \pm 10.4$ & $55.2 \pm 10.7 \pm 3.8$ \\
\hline$\gamma \gamma[\llbracket]$ & - & $<1.7(90 \%$ C.L. $)$ \\
\hline$K \ell^{+} \ell^{-}[5]$ & $0.0 \pm 0.3$ & $<0.6(90 \%$ C.L. $)$ \\
$K^{*} \ell^{+} \ell^{-}$ & $0.7 \pm 1.1$ & $<2.5(90 \%$ C.L.) \\
\hline
\end{tabular}

We have searched for $B^{+} \rightarrow K^{(*)+} \ell^{+} \ell^{-}$with $K^{*+} \rightarrow K_{S}^{0} \pi^{+}$and $B^{0} \rightarrow K^{(*) 0} \ell^{+} \ell^{-}$with $K^{* 0} \rightarrow$ $K^{+} \pi^{-}$. Not only does this analysis need to suppress background from continuum events, it also needs to suppress background from $B \bar{B}$ events. Major sources of $B \bar{B}$ background are events in which both $B$ mesons decay semileptonically or one decays to $J / \psi K^{(*)}$ or $\psi(2 \mathrm{~S}) K^{(*)}$ final states with $J / \psi \rightarrow \ell^{+} \ell^{-}$or $\psi(2 \mathrm{~S}) \rightarrow \ell^{+} \ell^{-}$. It is particularly important to suppress $B$ decays to charmonium since these have characteristics that are similar to the signal. The event yields are extracted with an unbinned likelihood fit in the $\Delta E-m_{E S}$ plane. The overall efficiencies for the different modes range between $6 \%$ for $K^{*+} \mu^{+} \mu^{-}$and $18 \%$ for $K^{+} e^{+} e^{-}$. No significant signal is observed but the limits (see Table 11) are close to SM calculations.

\section{RARE HADRONIC B DECAYS}

Rare hadronic decays have been searched for in two-body, quasi two-body and three-body modes. In the case of two-body decays, the following topologies are examined: $h^{+} h^{\prime-}, h^{+} \pi^{0}, K_{S}^{0} h^{+}$, $K_{S}^{0} \pi^{0}$ and $K_{S}^{0} K_{S}^{0}$, where $h$ represents a charged pion or kaon. Signal yields are extracted using an extended likelihood function taking all relevant topologies into account. The likelihood incorpo- 
rates information from event kinematics $(\Delta E$ and $\left.m_{E S}\right)$, event shape and energy flow via a Fisher discriminant, as well as mode-specific information like the Cherenkov angle for charged hadrons, the invariant mass for $K_{S}^{0} \rightarrow \pi^{+} \pi^{-}$candidates or the helicity angle. This analysis benefits from the excellent $\pi / K$ separation provided by the DIRC. The significance of the separation is greater than $4 \sigma$ up to laboratory momenta of $3 \mathrm{GeV} / c$ and is $2.5 \sigma$ at the highest momentum $(\sim 4.3 \mathrm{GeV} / c)$.

Signal yields and branching fractions are summarized in Table 2. The large rate for $K \pi$ final states as compared to $\pi \pi$ final states confirms the previous observation by the CLEO Collaboration. Since tree diagrams for $B^{0} \rightarrow K^{+} \pi^{-}$decays are Cabibbo-suppressed as compared to $B^{0} \rightarrow \pi^{+} \pi^{-}$ decays, the larger rate for $K \pi$ final states is a clear indication that gluonic penguin diagrams contribute significantly to the decay.

A host of quasi two-body decays were also studied. These involve $\omega, \eta, \eta^{\prime}$ or $\phi$ resonances and offer another window to study decays mediated by either or both tree and penguin decay amplitudes. For example, $B \rightarrow \omega \pi$ decays are dominated by tree decay amplitudes, whereas $B \rightarrow \phi K$ decays are dominated by gluonic penguin decay amplitudes. The later decay mode also holds the promise of a $\sin 2 \beta$ measurement via the timedependent asymmetry in $B^{0} \rightarrow \phi K_{S}^{0}$. Such a measurement is of interest to test the consistency of the CKM picture in two different decay mechanisms, $B^{0} \rightarrow J / \psi K_{s}^{0}$ involving $b \rightarrow c \bar{c} s$ transitions and $B^{0} \rightarrow \phi K_{S}^{0}$ involving $b \rightarrow s \bar{s} s$ transitions. $B \rightarrow \phi K^{0}$ and $B^{+} \rightarrow \phi K^{*+}$ decays were first observed by the $B A B A R$ Collaboration. A significant signal is also observed in $B^{+} \rightarrow \omega \pi^{+}$ decays, see Table 2 and Fig. 2.

Previous studies of $B \rightarrow \eta^{(\prime)} K^{(*)}$ decays reported a surprizingly large branching fraction. With a larger data sample, we confirm higher than expected rates for $B \rightarrow \eta K^{*}$ and $B \rightarrow \eta^{\prime} K$ decays, see Table 2 and Fig. 2. Since tree contributions are Cabibbo-suppressed one needs to invoke a large enhancement in the penguin contributions, possibly originating from interference between different penguin amplitudes $\left(g^{*} \rightarrow u \bar{u}\right.$ and $\left.g^{*} \rightarrow s \bar{s}\right)$.
Table 2

Summary of signal yields and branching fraction measurements for rare hadronic decays. The first and second uncertainties in the branching fractions are statistical and systematic, respectively. The uncertainty in the yield is statistical only.

\begin{tabular}{|c|c|c|}
\hline Mode & Signal yield & $\mathcal{B}\left(10^{-6}\right)$ \\
\hline$\pi^{+} \pi^{-}$《6 & $41 \pm 10$ & $4.1 \pm 1.0 \pm 0.7$ \\
\hline$K^{+} \pi^{-}$ & $169 \pm 17$ & $16.7 \pm 1.6 \pm 1.3$ \\
\hline$K^{+} K^{-}$ & $8.2_{-6.4}^{+7.8}$ & $<2.5$ (90\% C.L.) \\
\hline$\pi^{+} \pi^{0}$ & $37 \pm 14$ & $<9.6$ (90\% C.L.) \\
\hline$K^{+} \pi^{0}$ & $75 \pm 14$ & $10.8_{-1.9}^{+2.1} \pm 1.0$ \\
\hline$K^{0} \pi^{+}$ & $59_{-10}^{+11}$ & $18.2_{-3.0}^{+3.3} \pm 2.0$ \\
\hline $\bar{K}^{0} K^{+}$ & $-4.1_{-3.8}^{+4.5}$ & $<2.4$ (90\% C.L.) \\
\hline$K^{0} \pi^{0}$ & $17.9_{-5.8}^{+6.8}$ & $8.2_{-2.7}^{+3.1} \pm 1.2$ \\
\hline$K^{0} \bar{K}^{0}$ & $3.4_{-2.4}^{+3.4}$ & $<7.3$ (90\% C.L.) \\
\hline$\phi K^{+}[8]$ & $31.4_{-5.9}^{+6.7}$ & $7.7_{-1.4}^{+1.6} \pm 0.8$ \\
\hline$\phi K^{0}$ & $10.8_{-3.3}^{+4.1}$ & $8.1_{-2.5}^{+3.1} \pm 0.8$ \\
\hline$\phi K^{*+}$ & - & $9.7_{-3.4}^{+4.2} \pm 1.7$ \\
\hline$\phi K_{K}^{*}$ & $7.1_{-3.4}^{+4.3}$ & $12.8_{-6.1}^{+7.7} \pm 3.2$ \\
\hline$\phi K_{K^{0}}^{*+} \pi^{+}$ & $4.4_{-2.0}^{+2.7}$ & $8.0_{-3.7}^{+5.0} \pm 1.3$ \\
\hline$\phi K^{* 0}$ & $20.8_{-5.1}^{+5.9}$ & $8.7_{-2.1}^{+2.5} \pm 1.1$ \\
\hline$\phi \pi^{+}$ & $0.9_{-0.9}^{+2.1^{1}}$ & $<1.4$ (90\% C.L.) \\
\hline$\omega K^{+}$[9] & $6.4_{-4.4}^{+5.6}$ & $<4$ (90\% C.L.) \\
\hline$\omega K^{0}$ & $8.1_{-3.6}^{+4.6}$ & $<13$ (90\% C.L.) \\
\hline$\omega \pi^{+}$ & $27.6_{-7.7}^{+8.8}$ & $6.6_{-1.8}^{+2.1} \pm 0.7$ \\
\hline$\omega \pi^{0}$ & $-0.9_{-3.2}^{+5.0}$ & $<3$ (90\% C.L.) \\
\hline$\eta K^{* 0}$ & $20.5 \pm 6.0$ & $19.8_{-5.6}^{+6.5} \pm 1.7$ \\
\hline$\eta K^{*+}$ & $14.3 \pm 6.6$ & $<33.9$ (90\% C.L.) \\
\hline$\eta^{\prime} K^{+}[9]$ & - & $70 \pm 8 \pm 5$ \\
\hline$\eta_{n \pi \pi}^{\prime} \bar{K}^{+}$ & $49.5_{-7.3}^{+8.1}$ & $63_{-9}^{+10}$ \\
\hline$\eta_{\eta \gamma}^{\prime} K^{+}$ & $87.6_{-12.5}^{+13.4}$ & $80_{-11}^{+12}$ \\
\hline$\eta^{\prime} K^{0}$ & +12.0 & $42_{-11}^{+13} \pm 4$ \\
\hline$\eta_{\eta \pi \pi}^{\prime} K^{0}$ & $6.3_{-2.5}^{+3.3}$ & $28_{-11}^{+15}$ \\
\hline$\eta_{\eta \gamma}^{\prime} K^{0}$ & $20.8_{-6.5}^{+7.4}$ & $61_{-19}^{+22}$ \\
\hline$\eta^{\prime} \pi^{+}$ & - & $<12$ (90\% C.L.) \\
\hline$\eta_{\eta \pi \pi}^{\prime} \pi^{+}$ & $5.7_{-2.8}^{+3.8}$ & $7.1_{-3.5}^{+4.8}$ \\
\hline$\eta_{\eta \gamma}^{\prime} \pi^{+}$ & $-0.9_{-6.2}^{+7.8}$ & $-0.7_{-5.3}^{+6.7}$ \\
\hline \begin{tabular}{l|l|}
$a_{0}^{ \pm} \pi^{\mp}$ & 12 \\
\end{tabular} & $18.1_{-7.4}^{+8.7}$ & $<11.5$ (90\% C.L.) \\
\hline \begin{tabular}{l|l|l|}
$\rho^{ \pm} \pi^{\mp}$ & 13 \\
\end{tabular} & $89 \pm 16$ & $28.9 \pm 5.4 \pm 4.3$ \\
\hline$\rho^{0} \pi^{0}$ & $6.1 \pm 5.8$ & $<10.6$ (90\% C.L.) \\
\hline$K^{* 0} \pi^{+}$ & $34.8 \pm 7.6$ & $15.5 \pm 3.4 \pm 1.5$ \\
\hline
\end{tabular}



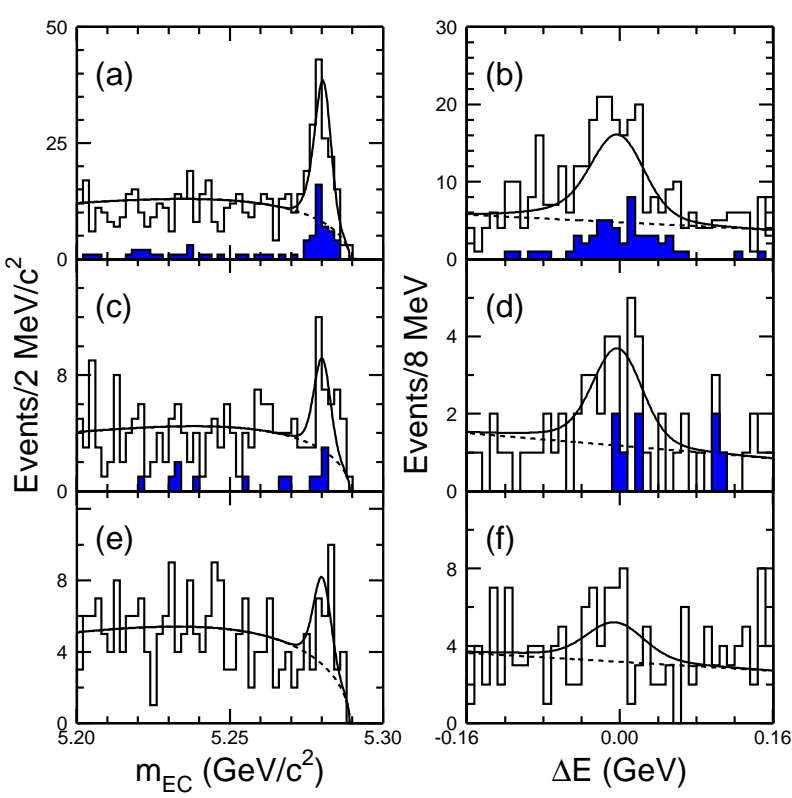

Figure 2. Distributions of $m_{E C}$ and $\Delta E$ for $(\mathrm{a}, \mathrm{b}) B^{+} \rightarrow \eta^{\prime} K^{+},(\mathrm{c}, \mathrm{d}) B^{0} \rightarrow \eta^{\prime} K^{0}$, and $(\mathrm{e}, \mathrm{f})$ $B^{+} \rightarrow \omega \pi^{+}$. The shaded area corresponds to $\eta^{\prime} \rightarrow \eta \pi \pi$. The solid curves represent the result of the likelihood function and the dashed curves correspond to the background contribution.

To further explore the large decay rate into $\eta^{\prime}$, we have performed a semi-inclusive reconstruction of the decay $B \rightarrow \eta^{\prime} X_{s}$, where $X_{s}$ represents a hadronic system with non-zero strangeness. The $\eta^{\prime}$ candidates are reconstructed in the $\eta \pi^{+} \pi^{-}$ channel (with $\eta \rightarrow \gamma \gamma$ ) and are required to have momentum $p^{*}>2 \mathrm{GeV} / c$ in the CMS to remove most of the $b \rightarrow c \rightarrow \eta^{\prime}$ background. The hadronic system is reconstructed in 16 different modes including one kaon and at most 3 pions (and no more than $1 \pi^{0}$ ). To discriminate between all combinations in a given event, the combination with the lowest value of $\chi^{2}=$ $\left(\frac{m_{E S}-m_{B}}{\sigma_{m_{E S}}}\right)^{2}+\left(\frac{\Delta E}{\sigma_{\Delta E}}\right)^{2}$ is selected. The rate for $B \rightarrow \eta^{\prime} X_{s}$ events with $p_{\eta^{\prime}}^{*}>2 \mathrm{GeV} / c$ is found to be $\left(6.8_{-1.0}^{+0.7} \pm 0.6_{-0.5}^{+0.0}\right) \times 10^{-4}$, where the first error is statistical, the second is systematic, and the third corresponds to the uncertainty in the contribution from $B \rightarrow \eta^{\prime} D^{(*) 0}$ decays. This measure- ment confirms the large rate previously measured by CLEO. Such a large rate is generally believed to be due to the anomalous coupling of the $\eta^{\prime}$ to two gluons.

We have also investigated $B$ decays to threebody final states. Particularly promising is the decay $B^{0} \rightarrow \pi^{+} \pi^{-} \pi^{0}$, which could provide a clean measurement of the CP-violating phase $\alpha$ via a Dalitz amplitude analysis. Due to the small expected signal yield, the current version of the analysis aims to determine the $B$ decay rates to $\rho^{ \pm}(770) \pi^{\mp}, \rho^{0}(770) \pi^{0}, \rho^{ \pm}(1450) \pi^{\mp}$, $\rho^{0}(1450) \pi^{0}$, (charged scalar) ${ }^{ \pm} \pi^{\mp}, f^{0} \pi^{0}$, and nonresonant $\pi^{+} \pi^{-} \pi^{0}$ final states. A significant signal is found only in the $B^{0} \rightarrow \rho^{ \pm}(770) \pi^{\mp}$ mode.

Also promising for the measurement of $\alpha$ is the decay $B^{0} \rightarrow a_{0}^{ \pm}(980) \pi^{\mp}$, which is expected to be free of main tree contributions. The analysis relies on a neural network algorithm to select $B^{0}$ decays to $a_{0}^{ \pm}(980) \pi^{\mp}$, with $a_{0}^{ \pm} \rightarrow \eta \pi^{ \pm}$and $\eta \rightarrow \gamma \gamma$. A likelihood fit using the neural network output, $\gamma \gamma$ mass, $m_{E S}$ and $\Delta E$ extracts a signal yield of $18.1_{-7.4}^{+8.7}$ events with a corresponding statistical significance of $3.7 \sigma$. The $\eta \pi^{ \pm}$invariant mass is not included in the fit because the parameters of the $a_{0}$ resonance are not well known.

Finally, we also measured the branching fraction for $B^{+}$decays into $K^{* 0} \pi^{+}$. Part of the interest in this process stems from the expectation that tree and penguin amplitudes have comparable magnitudes, which makes this decay an ideal candidate for the observation of direct $\mathrm{CP}$ violation, see Eq. 2. The measured yields and branching fractions for this and all other rare hadronic decays are summarized in Table 2.

\section{DIRECT CP VIOLATION}

We searched for CP violation in the decay of the flavor self-tagged modes listed in Table 3. The table shows the measured $\mathrm{CP}$-asymmetries as defined in Eq. 1. For the $B^{0} \rightarrow K^{+} \pi^{-}$decay mode, an on-resonance sample with an integrated luminosity of $30.4 \mathrm{fb}^{-1}$ is used 15]. In the case of the $B^{0} \rightarrow \rho^{ \pm} \pi^{\mp}$ mode, no tagging of the $B^{0}$ flavor is attempted and the asymmetry quoted in Table 3 corresponds to the asymmetry between the number of $\rho^{+} \pi^{-}$and $\rho^{-} \pi^{+}$events. No evi- 
Table 3

Summary of direct CP violation measurements. The first and second uncertainties are statistical and systematic, respectively.

\begin{tabular}{|c|c|}
\hline Mode & $A_{C P}$ \\
\hline$K^{*} \gamma[3]$ & $-0.044 \pm 0.076 \pm 0.012$ \\
\hline$K^{+} \pi^{-}$-15 & $-0.07 \pm 0.08 \pm 0.04$ \\
\hline$K^{+} \pi^{0}[6]$ & $0.00 \pm 0.18 \pm 0.04$ \\
\hline$K^{0} \pi^{+}$ & $-0.21 \pm 0.18 \pm 0.03$ \\
\hline$\phi K^{+}$ & $-0.05 \pm 0.20 \pm 0.03$ \\
\hline$\phi K^{*+}$ & $-0.43_{-0.30}^{+0.36} \pm 0.06$ \\
\hline$\phi K^{* 0}$ & $0.00 \pm 0.27 \pm 0.03$ \\
\hline$\omega \pi^{+} 16$ & $-0.01_{-0.31}^{+0.29} \pm 0.03$ \\
\hline$\eta^{\prime} K^{+}$-16" & $-0.11 \pm 0.11 \pm 0.02$ \\
\hline$\rho^{ \pm} \pi^{\mp} 13$ & $-0.04 \pm 0.18 \pm 0.02$ \\
\hline
\end{tabular}

dence for direct $\mathrm{CP}$ violation has been found but the precision is now better than $10 \%$ in some of the more abundant modes and, thus, these data become more useful in constraining models.

\section{SUMMARY AND OUTLOOK}

The large data sample accumulated at the SLAC PEP-II B Factory is already providing a substantial amount of interesting results. We are now sensitive to rare $B$ decays with branching fractions as low as $4 \times 10^{-6}$. A search for direct $\mathrm{CP}$ violation in radiative penguin and rare hadronic decays finds no evidence for $\mathrm{CP}$ violation in the decay process. It should be noted that some of the results presented here are preliminary.

PEP-II is expected to deliver an integrated luminosity of approximately $100 \mathrm{fb}^{-1}$ by the summer of 2002, which will provide a five-fold increase in statistics compared to the results reported here. This will allow many more rare processes to be studied.

\section{REFERENCES}

1. B. Aubert et al., Phys. Rev. Lett. 87, 091801 (2001); K. Abe et al., Phys. Rev. Lett. 87, 091802 (2001).

2. B. Aubert et al., SLAC-PUB-8569, to appear in Nucl. Instr. and Methods.
3. B. Aubert et al., SLAC-PUB-8952, hepex/0110065.

4. B. Aubert et al., Phys. Rev. Lett. 87, 241803 (2001).

5. B. Aubert et al., SLAC-PUB-8910, hepex/0107026.

6. B. Aubert et al., Phys. Rev. Lett. 87, 151802 (2001).

7. B. Aubert et al., SLAC-PUB-8978, hepex/0109005.

8. B. Aubert et al., Phys. Rev. Lett. 87, 151801 (2001).

9. B. Aubert et al., Phys. Rev. Lett. 87, 221802 (2001).

10. B. Aubert et al., SLAC-PUB-8914, hepex/0107037.

11. B. Aubert et al., SLAC-PUB-8979, hepex/0109034.

12. B. Aubert et al., SLAC-PUB-8930, hepex/0107075.

13. B. Aubert et al., SLAC-PUB-8926, hepex/0107058.

14. B. Aubert et al., SLAC-PUB-8981, hepex/0109007.

15. B. Aubert et al., SLAC-PUB-8929, hepex/0107074.

16. B. Aubert et al., SLAC-PUB-8980, hepex/0109006. 